Proceedings of the European Conference Physics of Magnetism 2011 (PM'11), Poznań, June 27-July 1, 2011

\title{
Non-Linear Thermal Current through Multilevel Quantum Dot Coupled to Ferromagnetic Electrodes
}

\author{
M. WiERZBiCKI AND R. ŚWiRKOWICZ \\ Faculty of Physics, Warsaw University of Technology, Koszykowa 75, 00-662 Warsaw, Poland
}

\begin{abstract}
We present studies of spin-dependent phenomena in the non-linear energy transport through a multilevel quantum dot/molecule in the Coulomb blockade regime. Calculations are performed within the framework of non-equilibrium Green function formalism based on the equation of motion. Thermal current flowing through the system due to temperature gradient significantly varies with gate voltage. It depends on relative orientation of magnetic moments in the electrodes, so in analogy to tunneling magnetoresistance the magnetothermal conductance is introduced, which describes the effect.
\end{abstract}

PACS: 73.23.Hk, 73.50.Lw, 85.80.Fi

\section{Introduction}

Recently, interplay between spin effects and thermoelectric phenomena in nanostructure systems have been intensively studied. Spin Seebeck effect has been observed for a ferromagnetic slab and spin voltage generated by a temperature gradient has been measured [1] A significant dependence of thermal coefficients on relative alignment of magnetic moments has been obtained for magnetic multilayer nanostructures with use of finite-element theory [2]. Thermoelectric properties of nanoscale systems containing quantum dots (QD) are strongly influenced both by quantum confinement and the Coulomb blockade effect, which lead to oscillations of thermal coefficients with gate voltage [3]. Additional fine structure due to discrete levels was found for small dots [4]. Spin effect in thermal transport through a QD attached to ferromagnetic leads was also studied [5-7]. Spin Seebeck effect and spin-dependent thermal efficiency, described by the spin-dependent figure of merit was discussed [5, 6].

To study transport phenomena in systems subjected to a considerable voltage and temperature gradient, a general approach, which allows to describe non-linear effects, is required. Thermal phenomena in the non-linear transport through QD were mainly investigated for non-magnetic systems, in which spin effects are irrelevant.

Here, we present studies of spin-dependent phenomena in the non-linear heat transport through a two-level $\mathrm{QD} /$ molecule in the Coulomb blockade regime, based on the non-equilibrium Green function formalism.

\section{Model}

We consider a nanoscopic system composed of a two-level QD/molecule attached to ferromagnetic leads. Electrical and heat currents flowing in the system due to bias voltage and temperature gradient can be expressed in a following way:

$$
\begin{aligned}
& I=\sum_{\sigma} I_{\sigma}=\frac{e}{\hbar} \sum_{\sigma} \int \frac{\mathrm{d} E}{2 \pi} T_{\sigma}(E)\left(f_{\mathrm{L} \sigma}-f_{\mathrm{R} \sigma}\right), \\
& I_{E}=\frac{1}{\hbar} \sum_{\sigma} \int \frac{\mathrm{d} E}{2 \pi} T_{\sigma}(E)\left(E-\mu_{0}+\frac{1}{2} e V\right)\left(f_{\mathrm{L}}-f_{\mathrm{R}}\right) .
\end{aligned}
$$

$f_{\beta}$ denotes here the Fermi-Dirac distribution function in the $\beta(=\mathrm{L}, \mathrm{R})$ electrode with electrochemical potential $\mu_{\mathrm{L}, \mathrm{R}}=\mu_{0} \pm \frac{1}{2} \mathrm{eV} . \mu_{0}$ corresponds to the Fermi energy of the system in equilibrium. Within the Green function approach the transmission $T_{\sigma}(E)$ in the spin channel $\sigma$ takes the form: $T_{\sigma}(E)=\sum_{j} \frac{\Gamma_{j \sigma}^{\mathrm{L}} \Gamma_{j \sigma}^{\mathrm{R}}}{\Gamma_{j \sigma}^{\mathrm{L}}+\Gamma_{j \sigma}^{\mathrm{R}}} \mathrm{i}\left(G_{j \sigma}^{\mathrm{r}}-G_{j \sigma}^{\mathrm{a}}\right)$. Here $G_{j \sigma}^{\mathrm{r}}\left(G_{j \sigma}^{\mathrm{a}}\right)$ denotes the retarded (advanced) Green function, whereas $\Gamma_{j \sigma}^{\beta}=\Gamma_{j}\left(1+\hat{\sigma} p_{\beta}\right)$ determines the spin-dependent coupling strength of the dot level $j$ with electrode $\beta, p_{\beta}$ is related to the lead's polarization and $\hat{\sigma}=1$ for spin index $\sigma=\uparrow$ or $\hat{\sigma}=-1$ for $\sigma=\downarrow$. $\Gamma_{j}$ denotes the coupling strength of the level $j$ with electrodes and is treated as a parameter independent of energy. Coupling strengths $\Gamma_{j}$ for two molecular levels $j=1,2$ can be different due to different spatial distribution of the corresponding wave functions. To describe such a dependence, we express $\Gamma_{j}$ in the form $\Gamma_{j}=\Gamma\left[1-(-1)^{j} Q\right]$. For $Q=0$ both levels 1 and 2 are equally coupled to electrodes, whereas for $0<Q<1$ one of the levels becomes weakly coupled.

The system under consideration is described by the Hamiltonian $H=H_{\mathrm{D}}+H_{\mathrm{e}}+H_{\mathrm{T}}$. The first term $H_{\mathrm{D}}$ corresponds to the dot and is taken in the form

$$
H_{\mathrm{D}}=\sum_{j \sigma} \varepsilon_{j} d_{j \sigma}^{+} d_{j \sigma}+\frac{1}{2} \sum_{i j \sigma \sigma^{\prime}} U_{i j} d_{i \sigma}^{+} d_{i \sigma} d_{j \sigma^{\prime}}^{+} d_{j \sigma^{\prime}},
$$

where $\varepsilon_{j}$ is the energy of the level $j, U_{j}$ and $U_{i j}$ describe intra and inter-level Coulomb correlations, respectively. $d_{j \sigma}^{+}\left(d_{j \sigma}\right)$ represents creation (annihilation) operator of electron in the state $j \sigma$. The term $H_{\mathrm{e}}$ in the main Hamiltonian corresponds to the non-interacting electrons in the leads and $H_{\mathrm{T}}$ describes tunneling effects between 
the dot and electrodes, and is taken in the standard form.

The non-equilibrium Green function formalism based on the equation of motion method is applied to investigate the electron and energy transport [8, 9]: the Green function $G_{j \sigma}^{\mathrm{r}}$ calculated within the HartreeFock approximation takes the form: $G_{j \sigma}^{\mathrm{r}}(E)=$ $\sum_{k=0}^{2} p_{k}\left(\frac{1-N_{j-\sigma}}{E-\varepsilon_{j}-A_{k}-\Sigma_{j}^{\mathrm{r}}}+\frac{N_{j-\sigma}}{E-\varepsilon_{j}-U_{j}-A_{k}-\Sigma_{j}^{\mathrm{r}}}\right)$. The summation is over possible configurations with level $l$, different from $j(l=-j)$ occupied by zero, one or two particles, respectively. $p_{k}$ denotes here the probability factor of a particular configuration $k$ and is expressed in terms of the average one-particle $N_{l \sigma}=\left\langle d_{l \sigma}^{+} d_{l \sigma}\right\rangle$ and two-particle $\left\langle N_{l-\sigma} N_{l \sigma}\right\rangle$ occupation numbers which are calculated in a self-consistent way. $A_{k}$ in the last equation denotes the sum of all interactions seen by the electron in the level $j$ due to other particles occupying the level $l$ in configuration $k$ and expressed in terms of $U_{j l}$ [7-9]. After determining the Green functions, one can calculate the transmission and study transport properties.

\section{Thermal current and thermal conductance. Numerical results}

The heat current $I_{E}$ is calculated according to formula (2) under the condition of vanishing charge current $I=0$. The coupling strengths of both levels are the same and are equal to $\Gamma=0.01 \mathrm{meV}$. Energy difference between the dot levels is assumed to be $\delta \varepsilon=0.6 \mathrm{meV}$. Positions of both levels can be coherently shifted with use of a gate voltage $V_{\mathrm{g}}$ starting from the energy $\varepsilon_{0}=-0.05 \mathrm{meV}$. The intra and inter-level correlation parameters are taken as: $U_{1}=U_{2}=2 \mathrm{meV}$ and $U_{12}=1 \mathrm{meV}$, respectively. In the presence of temperature gradient $\Delta T$, the temperature of the left electrode is increased by $\Delta T$, whereas it is fixed in the right electrode and equal to $k T_{\mathrm{R}}=0.02 \mathrm{meV}$. In such conditions the voltage is induced, which influences $I_{E}$. Calculations show that the heat current relatively weakly depends on the polarization factor. Thereby we present results only for the case with $p=0.5$.

In Fig. 1 the heat current and differential thermal conductance $\kappa=\mathrm{d} I_{E} / \mathrm{d}(k T)$ are depicted as a function of temperature gradient. As expected, $I_{E}$ monotonically increases with $\Delta T$, but it strongly depends on the gate voltage and the parameter $Q$. For $V_{\mathrm{g}}=0.06 \mathrm{meV}$ the level 1 with energy $\varepsilon_{0}=-0.05 \mathrm{meV}$ is active in the transport. In the region of small $\Delta T$ the heat current as well as thermal conductance $\kappa$ are enhanced for large $Q$ values (Fig. 1a and b). In such a situation, the level 1 is strongly coupled to electrodes which results in enhancement of transmission. With increase of $\Delta T$ the conductance $\kappa$ at first decreases. Then, $\kappa$ starts to increase, but now the lowest rate corresponds to large $Q$. At relatively high temperature the broadening of the Fermi-Dirac distribution function plays an important role, the levels start to overlap and the level 2 becomes active in the transport. Contribution to the transmission coefficient, coming from this level strongly depends on $Q$ and is suppressed for
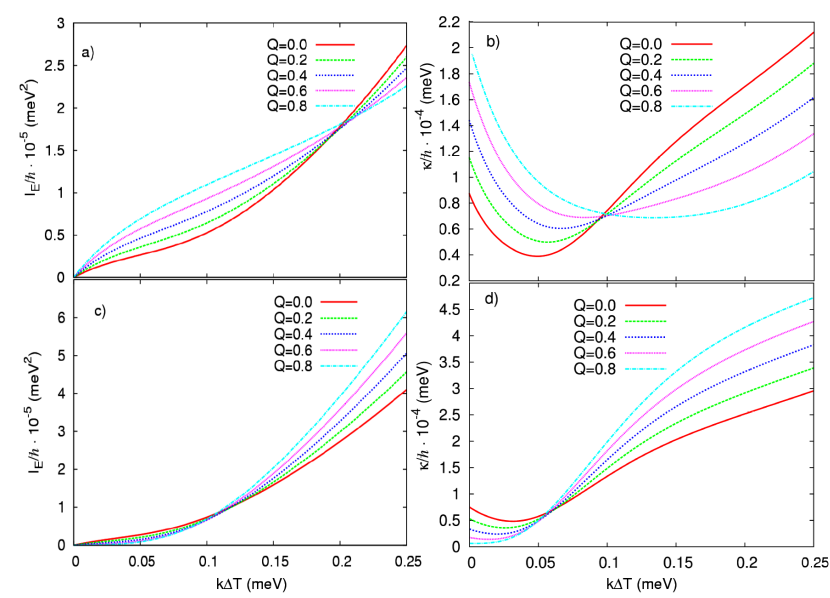

Fig. 1. Heat current (a, c) and thermal conductance (b, d) as a function of temperature difference for indicated values of $Q$ and two different gate voltages $V_{\mathrm{g}}=0.06 \mathrm{meV}(\mathrm{a}, \mathrm{b})$ and $V_{\mathrm{g}}=-1.56 \mathrm{meV}$. Parallel configuration with $p=0.5$. Other parameters are: $\varepsilon_{0}=-0.05 \mathrm{eV}, \delta \varepsilon=0.6 \mathrm{meV}, U=2 \mathrm{meV}, U_{12}=1 \mathrm{meV}$, $k T_{\mathrm{R}}=0.02 \mathrm{meV}$.

$Q=0.8$ due to weak coupling with electrodes. Therefore, in such a situation thermal conductance $\kappa$ does not change considerably with temperature. However, it increases significantly for low $Q$ values as both overlapping levels are strongly coupled with electrodes.

Quite different behavior can be observed for $V_{\mathrm{g}}=$ $-1.56 \mathrm{meV}$ (Fig. 1c and d). At low temperatures the level 1 is occupied and level 2 is mainly involved in the transport [9]. For larger $Q$ values this level becomes weakly coupled with electrodes, so the transmitted heat current is suppressed. The situation changes at high temperatures due to a strong broadening of the FermiDirac distribution function. Then, electrons can additionally tunnel through partly or singly occupied level 1 , as states with energy $\varepsilon_{0}$ or $\varepsilon_{0}+U$ overlap with the level 2 . Moreover, additional channels can open, as probabilities of particular one-particle and two-particle configurations strongly increase with temperature [9]. Though, for large $Q$, the level 2 is weakly coupled, the total, high temperature, transmission as well as the heat current increase. Let us note that due to all these contributions the heat current is much higher than in previous case with $V_{\mathrm{g}}=0.06 \mathrm{meV}$. A strong dependence of the heat current on the gate voltage is well illustrated in Fig. 2, where $I_{E}$ calculated for $Q=0.8$ is presented. It can be also seen that for $V_{\mathrm{g}}=-2.56 \mathrm{meV}$, corresponding to the Coulomb blockade regime, the energy transport is considerably suppressed in a wide temperature region.

Configuration of magnetic moments in electrodes strongly influences electron transport leading to tunneling magnetoresistance (TMR) effect. Similarly, the heat transport depends on the relative orientation of the leads' moments. So, one can calculate the heat current in the parallel configuration of the moments $I_{E}(\mathrm{P})$ as well as in 


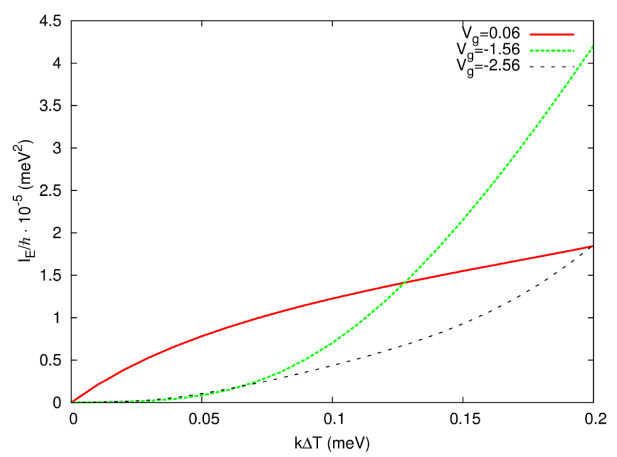

Fig. 2. Heat current as a function of temperature difference for indicated values of gate voltages $V_{\mathrm{g}}$ and $Q=0.8$. Other parameters are the same as in Fig. 1 .

the antiparallel one $I_{E}(A P)$ and define the magnetothermal conductance $\mathrm{MTC}=\left[I_{E}(\mathrm{P})-I_{E}(\mathrm{AP})\right] / I_{E}(\mathrm{AP})$.

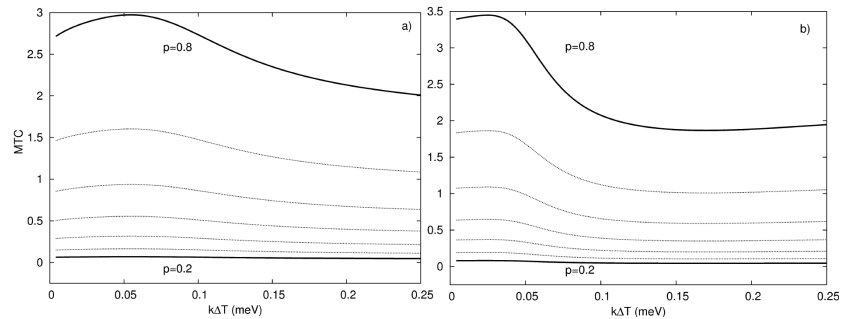

Fig. 3. Magnetothermal conductance as a function of temperature difference for several polarization factors calculated for $V_{\mathrm{g}}=0.06 \mathrm{meV}$ and $Q=0$ (a), $Q=$ 0.8 (b). Other parameters are the same as in Fig. 1.

Calculated MTC ratio as a function of temperature difference $\Delta T$ is presented in Fig. 3 for two values of parameter $Q$ and different polarization factors. MTC is positive in the whole temperature region, so the heat current, similarly to the charge current, is suppressed in antiparallel configuration. As presented in Fig. 3, MTC strongly changes with polarization factor and significantly increases with $p$. The ratio takes the largest values for small $\Delta T$, then it decreases. Let us note that MTC and therefore, spin effects are enhanced in systems with large $Q$, when the transmission through the lowest level increases. Thereby, for large $p$, in the parallel configuration, transmission in the majority spin channel is ad- ditionally enhanced, while the appropriate enhancement for the minority spin channel is negligible. With increase of temperature broadening of the Fermi-Dirac function becomes important which strongly suppresses the effect and MTC decreases more rapidly than for $Q=0$.

\section{Summary and conclusions}

Energy transport through a two-level QD (molecule) attached to ferromagnetic electrodes has been investigated in the non-linear regime. Thermal current flowing through the system due to temperature gradient, significantly varies with gate voltage. It can be suppressed in a wide temperature region for voltages corresponding to the Coulomb gap. The strongest effect can be observed for molecular junctions with one of the levels weakly coupled with electrodes. It has been found that energy transfer in such systems strongly depends on the relative orientation of magnetic moments in the electrodes and in analogy to TMR the MTC effect can be obtained. MTC ratio is positive indicating that, similarly to the charge transport, the energy transfer is suppressed in systems with antiparallel orientation of the moments.

\section{References}

[1] K. Uchida, S. Takahashi, K. Harii, J. Ieda, W. Koshibae, K. Ando, S. Maekawa, E. Saitoh, $\mathrm{Na}$ ture 455, 778 (2008).

[2] M. Hatami, G.E.W. Bauer, Q. Zhang, P.J. Kelly, Phys. Rev. B 79, 174426 (2009).

[3] J. Koch, F. von Oppen, Y. Oreg, E. Sela, Phys. Rev. B 70, 195107 (2004).

[4] M. Turek, K.A. Matveev, Phys. Rev. B 65, 115332 (2002).

[5] Y. Dubi, M. Di Ventra, Phys. Rev. B 79, 081302 (2009).

[6] R. Swirkowicz, M. Wierzbicki, J. Barnaś, Phys. Rev. B 80, 195409 (2009).

[7] M. Wierzbicki, R. Swirkowicz, Phys. Rev. B 82, 165334 (2010).

[8] Y.C. Chang, D.M.T. Kuo, Phys. Rev. B 77, 255412 (2008).

[9] M. Wierzbicki, R. Swirkowicz, J. Phys. Condens. Matter 22, 185302 (2010). 